\title{
On a $\rho$-orthogonality
}

\section{JACEK ChMieliński AND PAWEe WÓJCIK}

\section{Dedicated to Professor János Aczél on his 85th birthday}

\begin{abstract}
In a normed space we introduce an exact and approximate orthogonality relation connected with "norm derivatives" $\rho_{ \pm}^{\prime}$. We also consider classes of linear mappings preserving (exactly and approximately) this kind of orthogonality.
\end{abstract}

Mathematics Subject Classification (2010). Primary 46B20, 46C50; Secondary 39B82.

Keywords. Orthogonality, approximate orthogonality, orthogonality preserving mappings, norm derivative.

\section{Orthogonality}

Let $(X,\|\cdot\|)$ be a normed space over $\mathbb{K} \in\{\mathbb{R}, \mathbb{C}\}$. If the norm comes from an inner product $\langle\cdot \mid \cdot\rangle$, there is one natural orthogonality relation: $x \perp y \Leftrightarrow\langle x \mid y\rangle=$ 0 . In the general case, there are several notions of orthogonality and one of the most outstanding ones is the definition introduced by Birkhoff [4] (cf. also James [15]). For $x, y \in X$ we define:

$$
x \perp_{\mathrm{B}} y \quad \Longleftrightarrow \forall \lambda \in \mathbb{K}: \quad\|x+\lambda y\| \geq\|x\| .
$$

G. Lumer [17] and J.R. Giles [13] (cf. also [12]) proved that in a normed space $X$ there always exists a mapping $[\cdot \mid \cdot]: X \times X \rightarrow \mathbb{K}$ satisfying the following properties:

- $[\lambda x+\mu y \mid z]=\lambda[x \mid z]+\mu[y \mid z], \quad x, y, z \in X, \lambda, \mu \in \mathbb{K}$;

- $[x \mid \lambda y]=\bar{\lambda}[x \mid y], \quad x, y \in X, \lambda \in \mathbb{K}$;

- $|[x \mid y]| \leq\|x\| \cdot\|y\|, \quad x, y \in X$

and

- $[x \mid x]=\|x\|^{2}, \quad x \in X$.

Such a mapping is called a semi-inner-product (s.i.p.) in $X$ (generating the norm $\|\cdot\|)$. There may exist infinitely many different semi-inner-products in 
$X$. There is a unique one if and only if $X$ is smooth (i.e., there is a unique supporting hyperplane at each point of the unit sphere $S$ or, equivalently, the norm is Gâteaux differentiable on $S$-cf. [10,12]). If $X$ is an inner product space, the only s.i.p. on $X$ is the inner-product itself.

The semi-orthogonality of vectors $x$ and $y$ in $X$ (with respect to a given semi-inner-product) is defined as follows:

$$
x \perp_{\mathrm{s}} y \Longleftrightarrow[y \mid x]=0 .
$$

Of course, in an inner product space we have $\perp_{\mathrm{B}}=\perp_{\mathrm{s}}=\perp$.

From now on, we assume that the considered normed spaces are real and we define norm derivatives by:

$$
\rho_{ \pm}^{\prime}(x, y):=\lim _{t \rightarrow 0^{ \pm}} \frac{\|x+t y\|^{2}-\|x\|^{2}}{2 t}, \quad x, y \in X .
$$

The convexity of the norm yields that the above definition is meaningful. The mappings $\rho_{+}^{\prime}$ and $\rho_{-}^{\prime}$ are sometimes called the superior and inferior semi-innerproducts and their following properties, which will be useful in the present note, can be found, e.g., in $[2,3,12]$.

- There is always $\rho_{-}^{\prime} \leq \rho_{+}^{\prime}$;

- $\rho_{+}^{\prime}=\rho_{-}^{\prime}$ if and only if $X$ is smooth;

- for all $x, y \in X$ one has $\rho_{ \pm}^{\prime}(-x, y)=-\rho_{\mp}^{\prime}(x, y)$;

- the mappings $\rho_{ \pm}^{\prime}$ are continuous with respect to the second variable;

- for all $x, y \in X, \alpha \in \mathbb{R}$ :

$$
\rho_{ \pm}^{\prime}(x, \alpha x+y)=\alpha\|x\|^{2}+\rho_{ \pm}^{\prime}(x, y) ;
$$

- if $[\cdot \mid \cdot]$ is a given semi-inner-product in $X$, then

$$
\rho_{ \pm}^{\prime}(x, y)=\lim _{t \rightarrow 0^{ \pm}}[y \mid x+t y], \quad x, y \in X
$$

and moreover, if $X$ is smooth, then

$$
\rho_{ \pm}^{\prime}(x, y)=[y \mid x], \quad x, y \in X .
$$

The notion of M-semi-inner-product and its properties which follows have been given by Miličić [18] (cf. also [12]):

$$
\langle y \mid x\rangle_{g}:=\frac{1}{2}\left(\rho_{+}^{\prime}(x, y)+\rho_{-}^{\prime}(x, y)\right) .
$$

As a consequence of (1) we have

$$
\langle\alpha x+y \mid x\rangle_{g}=\alpha\|x\|^{2}+\langle y \mid x\rangle_{g} .
$$

A normed space $X$ is called semi-smooth if the M-semi-inner-product is additive with respect to the first variable, i.e.,

$$
\langle x+y \mid z\rangle_{g}=\langle x \mid z\rangle_{g}+\langle y \mid z\rangle_{g}, \quad x, y, z \in X .
$$


Each smooth space is semi-smooth in the above sense but not conversely $\left(l^{1}\right.$ is a suitable example). If $X$ is a real semi-smooth space, then the M-semi-innerproduct is a semi-inner-product in the sense of Lumer-Giles.

Now we can define orthogonality relations related to $\rho_{ \pm}^{\prime}$ (cf. $\left.[1,2,18]\right)$ :

$$
\begin{aligned}
& x \perp_{\rho_{+}} y \Longleftrightarrow \rho_{+}^{\prime}(x, y)=0 ; \\
& x \perp_{\rho_{-}} y \Longleftrightarrow \rho_{-}^{\prime}(x, y)=0
\end{aligned}
$$

and

$$
x \perp_{\rho} y \Longleftrightarrow\langle y \mid x\rangle_{g}=0 \Longleftrightarrow \rho_{+}^{\prime}(x, y)+\rho_{-}^{\prime}(x, y)=0 .
$$

It is obvious that for a real inner product space all the above relations coincide with the standard orthogonality given by the inner product, i.e., $\perp_{\rho}=\perp_{\rho_{+}}=$ $\perp_{\rho_{-}}=\perp$.

The relations $\perp_{\rho_{+}}, \perp_{\rho_{-}}$and $\perp_{\rho}$ are generally (unless $X$ is smooth) incomparable.

Theorem 1. In a real normed space $X$ the following conditions are equivalent:
(a) $\perp_{\rho_{+}} \subset \perp_{\rho_{-}}$
(b) $\perp_{\rho_{+}} \supset \perp_{\rho_{-}}($
(c) $\perp_{\rho_{+}}=\perp_{\rho_{-}}$
(d) $\perp_{\rho_{+}} \subset \perp_{\rho}$
$(e) \perp_{\rho_{+}} \supset \perp_{\rho}$
(f) $\perp_{\rho_{+}}=\perp_{\rho}$
(g) $\perp_{\rho_{-}} \subset \perp_{\rho}$
$(h) \perp_{\rho_{-}} \supset \perp_{\rho}$
(i) $\perp_{\rho_{-}}=\perp_{\rho}$
(j) $\rho_{+}^{\prime}=\rho_{-}^{\prime}$
(k)X is smooth.

Proof. We will show that conditions (a), (c) and (j) are equivalent. Obviously $(\mathrm{j}) \Rightarrow(\mathrm{c}) \Rightarrow(\mathrm{a})$. Suppose (a) and let $x, y \in X$ (we may assume $x \neq 0$, otherwise (j) holds trivially). From (1) we have for $z:=-\frac{\rho_{+}^{\prime}(x, y)}{\|x\|^{2}} x+y$

$$
\rho_{+}^{\prime}(x, z)=-\frac{\rho_{+}^{\prime}(x, y)}{\|x\|^{2}}\|x\|^{2}+\rho_{+}^{\prime}(x, y)=0,
$$

i.e., $x \perp_{\rho_{+}} z$. Hence, from (a), $x \perp_{\rho_{-}} z$, i.e.,

$$
\rho_{-}^{\prime}\left(x,-\frac{\rho_{+}^{\prime}(x, y)}{\|x\|^{2}} x+y\right)=0 .
$$

Using (1) again we get from the above that $\rho_{+}^{\prime}(x, y)=\rho_{-}^{\prime}(x, y)$, which proves (j).

We also have obviously $(\mathrm{j}) \Rightarrow(\mathrm{c}) \Rightarrow(\mathrm{b})$ and the proof of $(\mathrm{b}) \Rightarrow(\mathrm{j})$ is similar to the above one. Thus we have proved $(\mathrm{a}) \Leftrightarrow(\mathrm{b}) \Leftrightarrow(\mathrm{c}) \Leftrightarrow(\mathrm{j})$.

In the same manner, one can prove $(\mathrm{d}) \Leftrightarrow(\mathrm{e}) \Leftrightarrow(\mathrm{f}) \Leftrightarrow(\mathrm{j})$ and $(\mathrm{g}) \Leftrightarrow$ $(\mathrm{h}) \Leftrightarrow(\mathrm{i}) \Leftrightarrow(\mathrm{j})$.

The equivalence $(\mathrm{j}) \Leftrightarrow(\mathrm{k})$ is known. 
In a real normed space $X$, we have for arbitrary $x, y \in X$ (cf. [3, 12]):

$$
\rho_{-}^{\prime}(x, y) \leq 0 \leq \rho_{+}^{\prime}(x, y) \Longleftrightarrow x \perp_{\mathrm{B}} y \text {. }
$$

It yields $\perp_{\rho} \subset \perp_{\mathrm{B}}$ and, if $X$ is smooth, then also $\perp_{\rho}=\perp_{\mathrm{B}}$.

The following result establishes the connection between $\perp_{\rho}$ and $\perp_{\mathrm{s}}$.

Theorem 2. Let $X$ be a real normed space and let $[\cdot \mid \cdot]$ be a given semi-innerproduct in $X$. Then the following conditions are equivalent:

(a) $\perp_{\rho} \subset \perp_{\mathrm{s}}$;

(b) $\perp_{\rho} \supset \perp_{\mathrm{s}}$;

(c) $\perp_{\rho}=\perp_{\mathrm{s}}$;

(d) $\langle\cdot \mid \cdot\rangle_{g}=[\cdot \mid \cdot] \cdot$

Proof. Implications (d) $\Rightarrow(\mathrm{a}),(\mathrm{d}) \Rightarrow(\mathrm{b}),(\mathrm{d}) \Rightarrow(\mathrm{c}),(\mathrm{c}) \Rightarrow(\mathrm{a}),(\mathrm{c}) \Rightarrow(\mathrm{b})$ are obvious. We show $(\mathrm{a}) \Rightarrow(\mathrm{d})$. Let $x, y \in X$ and $x \neq 0$ (for $x=0$ the assertion holds trivially). Using (4) we have for $z:=-\frac{\langle y \mid x\rangle_{g}}{\|x\|^{2}} x+y$

$$
\langle z \mid x\rangle_{g}=-\frac{\langle y \mid x\rangle_{g}}{\|x\|^{2}}\|x\|^{2}+\langle y \mid x\rangle_{g}=0
$$

i.e., $x \perp_{\rho} z$. Thus, from (a), $x \perp_{\mathrm{s}} z$, which means

$$
0=[z \mid x]=-\frac{\langle y \mid x\rangle_{g}}{\|x\|^{2}}[x \mid x]+[y \mid x]=-\langle y \mid x\rangle_{g}+[y \mid x],
$$

hence $\langle y \mid x\rangle_{g}=[y \mid x]$.

Implication $(\mathrm{b}) \Rightarrow(\mathrm{d})$ can be proved similarly.

Note that (d) yields, in particular, that $\langle\cdot \mid \cdot\rangle_{g}$ is additive with respect to the first variable, thus each of the (equivalent) conditions (a)-(d) implies the semi-smoothness of $X$. For a non-semi-smooth norm, the orthogonalities $\perp_{\rho}$ and $\perp_{\mathrm{s}}$ are incomparable.

\section{Approximate orthogonality}

Our aim is to discuss approximate orthogonality relations. In an inner product space, it is natural to consider the approximate orthogonality ( $\varepsilon$-orthogonality) defined by:

$$
x \perp^{\varepsilon} y \Longleftrightarrow|\langle x \mid y\rangle| \leq \varepsilon\|x\|\|y\|
$$

with a given $\varepsilon \in[0,1)$. The above notion can be easily adapted to the case of a normed space with a given semi-inner-product:

$$
x \perp_{\mathrm{s}}^{\varepsilon} y \Longleftrightarrow|[y \mid x]| \leq \varepsilon\|x\|\|y\| .
$$

An approximate Birkhoff orthogonality was given by Dragomir [11]:

$$
x \frac{\perp_{\varepsilon}}{\mathrm{B}} y \Longleftrightarrow \forall \lambda \in \mathbb{K}:\|x+\lambda y\| \geq(1-\varepsilon)\|x\|
$$


and an alternative (generally not equivalent) definition was given by Chmieliński [6]:

$$
x \perp_{\mathrm{B}}^{\varepsilon} y \Longleftrightarrow \forall \lambda \in \mathbb{K}:\|x+\lambda y\|^{2} \geq\|x\|^{2}-2 \varepsilon\|x\|\|\lambda y\| .
$$

Obviously, for $\varepsilon=0$ the above approximate orthogonalities coincide with the related exact orthogonalities.

Now, we introduce the notion of an approximate $\rho_{ \pm^{-}}$and $\rho$-orthogonality. For an $\varepsilon \in[0,1)$ we define:

$$
\begin{aligned}
& x \perp_{\rho_{+}}^{\varepsilon} y \Longleftrightarrow\left|\rho_{+}^{\prime}(x, y)\right| \leq \varepsilon\|x\|\|y\|, \\
& x \perp_{\rho_{-}}^{\varepsilon} y \Longleftrightarrow\left|\rho_{-}^{\prime}(x, y)\right| \leq \varepsilon\|x\|\|y\|
\end{aligned}
$$

and

$$
x \perp_{\rho}^{\varepsilon} y \Longleftrightarrow\left|\langle y \mid x\rangle_{g}\right| \leq \varepsilon\|x\|\|y\| \Longleftrightarrow\left|\rho_{+}^{\prime}(x, y)+\rho_{-}^{\prime}(x, y)\right| \leq 2 \varepsilon\|x\|\|y\| .
$$

It is easy to see that $x \perp_{\rho_{+}}^{\varepsilon} y \Rightarrow-x \perp_{\rho_{-}}^{\varepsilon} y$ and $x \perp_{\rho_{-}}^{\varepsilon} y \Rightarrow-x \perp_{\rho_{+}}^{\varepsilon} y$. Moreover, if $x \perp_{\rho_{+}}^{\varepsilon} y$ and $x \perp_{\rho_{-}}^{\varepsilon} y$, then $x \perp_{\rho}^{\varepsilon} y$. Obviously, if the norm in $X$ comes from an inner product, then $\perp_{\rho_{+}}^{\varepsilon}=\perp_{\rho_{-}}^{\varepsilon}=\perp_{\rho}^{\varepsilon}=\perp^{\varepsilon}$.

Theorem 3. For an arbitrary real normed space $X$ and $\varepsilon \in[0,1)$ we have

$$
\perp_{\rho}^{\varepsilon} \subset \perp_{\mathrm{B}}^{\varepsilon}
$$

Proof. Let $x, y \in X$ be non-zero vectors and assume that $x \perp_{\rho}^{\varepsilon} y$. Then

$$
-2 \varepsilon\|x\|\|y\| \leq \rho_{+}^{\prime}(x, y)+\rho_{-}^{\prime}(x, y) \leq 2 \varepsilon\|x\|\|y\| .
$$

Since $\rho_{-}^{\prime} \leq \rho_{+}^{\prime}$, we get from the above

$$
2 \rho_{-}^{\prime}(x, y) \leq 2 \varepsilon\|x\|\|y\|
$$

hence

$$
\lim _{t \rightarrow 0^{-}} \frac{\|x+t y\|^{2}-\|x\|^{2}}{t} \leq 2 \varepsilon\|x\|\|y\| .
$$

For a fixed $\gamma \in(0,1)$, we get

$$
\lim _{t \rightarrow 0^{-}} \frac{\|x+t y\|^{2}-\|x\|^{2}}{t}<2(\varepsilon+\gamma)\|x\|\|y\| .
$$

It follows that, for some $\delta_{1}<0$, one has

$$
\frac{\|x+t y\|^{2}-\|x\|^{2}}{t}<2(\varepsilon+\gamma)\|x\|\|y\|, \quad t \in\left[\delta_{1}, 0\right) .
$$

Equivalently

$$
\|x\|^{2}<\|x+t y\|^{2}+2(\varepsilon+\gamma)\|x\|\|t y\|, \quad t \in\left[\delta_{1}, 0\right) .
$$

Similarly, from $\rho_{-}^{\prime} \leq \rho_{+}^{\prime}$ and (6) one has

$$
-2 \varepsilon\|x\|\|y\| \leq 2 \rho_{+}^{\prime}(x, y)
$$


hence

$$
-2 \varepsilon\|x\|\|y\| \leq \lim _{t \rightarrow 0^{+}} \frac{\|x+t y\|^{2}-\|x\|^{2}}{t}
$$

and consequently

$$
-2(\varepsilon+\gamma)\|x\|\|y\|<\lim _{t \rightarrow 0^{+}} \frac{\|x+t y\|^{2}-\|x\|^{2}}{t} .
$$

It follows that, for some $\delta_{2}>0$, one has

$$
-2(\varepsilon+\gamma)\|x\|\|y\|<\frac{\|x+t y\|^{2}-\|x\|^{2}}{t}, \quad t \in\left(0, \delta_{2}\right]
$$

and equivalently

$$
\|x\|^{2}<\|x+t y\|^{2}+2(\varepsilon+\gamma)\|x\|\|t y\|, \quad t \in\left(0, \delta_{2}\right] .
$$

Let $\varphi(t):=\|x+t y\|^{2}+2(\varepsilon+\gamma)\|x\|\|t y\|$ for $t \in \mathbb{R}$. The mapping $\varphi: \mathbb{R} \rightarrow \mathbb{R}$ is convex and it follows from (7) and (8) that

$$
\varphi(0)<\varphi(t), \quad t \in\left[\delta_{1}, 0\right) \cup\left(0, \delta_{2}\right] .
$$

The convexity of $\varphi$ yields

$$
\varphi(0)<\varphi(t), \quad t \in \mathbb{R} \backslash\{0\},
$$

i.e.,

$$
\|x\|^{2}<\|x+t y\|^{2}+2(\varepsilon+\gamma)\|x\|\|t y\|, \quad t \in \mathbb{R} \backslash\{0\} .
$$

Fix $\lambda \neq 0$. From the above we have

$$
\|x\|^{2}<\|x+\lambda y\|^{2}+2(\varepsilon+\gamma)\|x\|\|\lambda y\|
$$

hence, on letting $\gamma \rightarrow 0^{+}$,

$$
\|x\|^{2} \leq\|x+\lambda y\|^{2}+2 \varepsilon\|x\|\|\lambda y\| .
$$

Obviously, the above inequality holds true also for $\lambda=0$ and hence we get $x \perp_{\mathrm{B}}^{\varepsilon} y$.

It has been proved (cf. [6]) that for an arbitrary semi-inner-product in $X$, $\perp_{\mathrm{s}}^{\varepsilon} \subset \perp_{\mathrm{B}}^{\varepsilon}$. Moreover, if the norm in $X$ is smooth, then (for the unique s.i.p. in that case) $\perp_{\mathrm{s}}^{\varepsilon}=\perp_{\mathrm{B}}^{\varepsilon}$. But generally, the equality $\perp_{\mathrm{s}}^{\varepsilon}=\perp_{\mathrm{B}}^{\varepsilon}$ (for all s.i.p.) does not hold (cf. [6, Example 3.1]).

It follows from (3) that in a real smooth space $X$

$$
\left|\rho_{+}^{\prime}(x, y)+\rho_{-}^{\prime}(x, y)\right|=2|[y \mid x]|, \quad x, y \in X
$$

hence $\perp_{\rho}^{\varepsilon}=\perp_{s}^{\varepsilon}$. Thus, the following assertion holds true.

Theorem 4. If $X$ is a real normed, smooth space and $\varepsilon \in[0,1)$, then

$$
\perp_{\rho}^{\varepsilon}=\perp_{\mathrm{B}}^{\varepsilon} .
$$

For non-smooth spaces, the approximate orthogonalities $\perp_{\rho}^{\varepsilon}$ and $\perp_{\mathrm{B}}^{\varepsilon}$ may not coincide. 
Example 5. Consider the space $\mathbb{R}^{3}$ with the norm $\left\|\left(x_{1}, x_{2}, x_{3}\right)\right\|=\left|x_{1}\right|+\left|x_{2}\right|+$ $\left|x_{3}\right|$. Let $\varepsilon \in\left[0, \frac{\sqrt{3}-1}{2}\right)$ and let $x=(1,0,0), y=(1,1,2 \varepsilon)$. Then, for an arbitrary $\lambda \in \mathbb{R}:$

$$
\|x+\lambda y\|=|1+\lambda|+|\lambda|+|2 \lambda \varepsilon| \geq 1=\|x\|,
$$

i.e., $x \perp_{\mathrm{B}} y$. On the other hand, we have

$$
\rho_{+}^{\prime}(x, y)=2+2 \varepsilon \quad \text { and } \quad \rho_{-}^{\prime}(x, y)=-2 \varepsilon,
$$

hence

$$
\left|\rho_{+}^{\prime}(x, y)+\rho_{-}^{\prime}(x, y)\right|=2>4 \varepsilon^{2}+4 \varepsilon=2 \varepsilon\|x\|\|y\| .
$$

Thus, for any $\varepsilon \in\left[0, \frac{\sqrt{3}-1}{2}\right.$ ) we have $x \perp_{\mathrm{B}}^{\varepsilon} y$ but not $x \perp_{\rho}^{\varepsilon} y$.

It is known (cf. $[2,12])$ that the equality of the considered orthogonality relations $\perp_{\rho}=\perp_{\mathrm{B}}$ yields the smoothness of the norm. We ask whether the smoothness of $X$ also results from $\perp_{\rho}^{\varepsilon}=\perp_{\mathrm{B}}^{\varepsilon}$ with some $\varepsilon \in(0,1)$.

\section{Orthogonality preserving mappings}

It has been proved by Koldobsky [16] (for real spaces) and Blanco and Turnšek [5] (for real and complex ones) that a linear mapping $f: X \rightarrow X$ preserving the Birkhoff orthogonality, i.e., satisfying

$$
x \perp_{\mathrm{B}} y \Longrightarrow f x \perp_{\mathrm{B}} f y, \quad x, y \in X
$$

has to be a similarity (scalar multiple of an isometry). The same assertion can also be derived for linear mappings preserving semi-orthogonality, i.e., satisfying

$$
x \perp_{\mathrm{s}} y \Longrightarrow f x \perp_{\mathrm{s}} f y, \quad x, y \in X
$$

with respect to some semi-inner-product in $X$ (cf. [5, Remark 3.2]).

Now, let us consider linear mappings $f: X \rightarrow X$ that preserve the $\perp_{\rho}$ $\left(\perp_{\rho_{ \pm}}\right)$orthogonality:

$$
\begin{gathered}
x \perp_{\rho_{+}} y \Longrightarrow f x \perp_{\rho_{+}} f y, \quad x, y \in X, \\
x \perp_{\rho_{-}} y \Longrightarrow f x \perp_{\rho_{-}} f y, \quad x, y \in X, \\
x \perp_{\rho} y \Longrightarrow f x \perp_{\rho} f y, \quad x, y \in X .
\end{gathered}
$$

The result of Blanco and Turnšek yields that if $X$ is a real semi-smooth space (hence an M-semi-inner-product is a semi-inner-product), then a linear mapping preserving $\rho$-orthogonality has to be a similarity.

We have also the following characterization of $\rho_{ \pm}$-orthogonality preserving mappings. 
Theorem 5. Let $X$ be a real normed space, $f: X \rightarrow X$ a nonzero, linear mapping. Then, the following conditions are equivalent:

(a) $f$ preserves $\rho_{+}$-orthogonality;

(b) $f$ preserves $\rho_{-}$-orthogonality;

(c) $\|f x\|=\|f\| \cdot\|x\|, \quad x \in X$;

(d) $\rho_{+}^{\prime}(f x, f y)=\|f\|^{2} \cdot \rho_{+}^{\prime}(x, y), \quad x, y \in X$;

(e) $\quad \rho_{-}^{\prime}(f x, f y)=\|f\|^{2} \cdot \rho_{-}^{\prime}(x, y), \quad x, y \in X$;

(f) $\langle f x \mid f y\rangle_{g}=\|f\|^{2} \cdot\langle x \mid y\rangle_{g}, \quad x, y \in X$.

Moreover, each of the above conditions implies

(g) $f$ preserves $\rho$-orthogonality.

Proof. First, we prove $(\mathrm{a}) \Leftrightarrow(\mathrm{b})$. Suppose that $f$ preserves $\rho_{+}$-orthogonality. Let $x, y \in X$ be such that $x \perp_{\rho_{-}} y$. Thus, $\rho_{+}^{\prime}(-x, y)=-\rho_{-}^{\prime}(x, y)=0$, i.e., $-x \perp_{\rho_{+}} y$. Since $f$ preserves $\rho_{+}$-orthogonality, we have $-f x \perp_{\rho_{+}} f y$ which yields $f x \perp_{\rho_{-}} f y$. The proof of the converse implication $(\mathrm{b}) \Rightarrow(\mathrm{a})$ is similar.

Now, we prove that (a) and (b) yield (c). Let $x, y \in X, x \neq 0$, be such that $x \perp_{\mathrm{B}} y$. From $(5)$ we have $\rho_{-}^{\prime}(x, y) \leq 0 \leq \rho_{+}^{\prime}(x, y)$ and hence

$$
\frac{\|f x\|^{2}}{\|x\|^{2}} \rho_{-}^{\prime}(x, y) \leq 0 \leq \frac{\|f x\|^{2}}{\|x\|^{2}} \rho_{+}^{\prime}(x, y) .
$$

We have

$$
\rho_{+}^{\prime}\left(x,-\frac{\rho_{+}^{\prime}(x, y)}{\|x\|^{2}} x+y\right)=-\frac{\rho_{+}^{\prime}(x, y)}{\|x\|^{2}}\|x\|^{2}+\rho_{+}^{\prime}(x, y)=0,
$$

i.e., $x \perp_{\rho_{+}}\left(-\frac{\rho_{+}^{\prime}(x, y)}{\|x\|^{2}} x+y\right)$. Now, (a) implies

$$
f x \perp_{\rho_{+}}\left(-\frac{\rho_{+}^{\prime}(x, y)}{\|x\|^{2}} f x+f y\right)
$$

hence

$$
0=\rho_{+}^{\prime}\left(f x,-\frac{\rho_{+}^{\prime}(x, y)}{\|x\|^{2}} f x+f y\right)=-\frac{\rho_{+}^{\prime}(x, y)}{\|x\|^{2}}\|f x\|^{2}+\rho_{+}^{\prime}(f x, f y) .
$$

It yields $\rho_{+}^{\prime}(f x, f y)=\frac{\|f x\|^{2}}{\|x\|^{2}} \rho_{+}^{\prime}(x, y)$ and similarly, (b) yields $\rho_{-}^{\prime}(f x, f y)=$ $\frac{\|f x\|^{2}}{\|x\|^{2}} \rho_{-}^{\prime}(x, y)$. Thus, inequalities (9) take the form

$$
\rho_{-}^{\prime}(f x, f y) \leq 0 \leq \rho_{+}(f x, f y)
$$

which gives $f x \perp_{\mathrm{B}} f y$. Hence, $f$ preserves Birkhoff orthogonality, i.e., $f$ is a similarity and (c) holds true. 
Let us show $(\mathrm{c}) \Rightarrow(\mathrm{d})$ and $(\mathrm{c}) \Rightarrow(\mathrm{e})$. Let $x, y \in X$ (without loss of generality we may assume $x \neq 0)$. We have from (c)

$$
\begin{aligned}
\rho_{ \pm}^{\prime}(x, y) & =\lim _{t \rightarrow 0^{ \pm}} \frac{\|x+t y\|^{2}-\|x\|^{2}}{2 t}=\frac{1}{\|f\|^{2}} \lim _{t \rightarrow 0^{ \pm}} \frac{\|f x+t f y\|^{2}-\|f x\|^{2}}{2 t} \\
& =\frac{1}{\|f\|^{2}} \rho_{ \pm}^{\prime}(f x, f y)
\end{aligned}
$$

and (d) and (e) follows. Implications $(\mathrm{d}) \Rightarrow(\mathrm{a})$ and $(\mathrm{e}) \Rightarrow(\mathrm{b})$ are obvious. Therefore, conditions (a)-(e) are equivalent. Condition (f) follows easily from (d) and (e) and, conversely, assuming (f) and taking $y=x$, one gets $\|f x\|^{2}=\|f\|^{2} \cdot\|x\|^{2}$ hence (c) follows.

Obviously, $(f)$ implies (g).

If $X$ is smooth, then we immediately have $(\mathrm{g}) \Rightarrow(\mathrm{a})$ and all the conditions (a)-(g) are equivalent. But smoothness is not necessary for that.

Example 7. Let $X$ be $\mathbb{R}^{2}$ with the norm $\|(x, y)\|=|x|+|y|$ and let $f: X \rightarrow X$ be a linear (hence continuous) mapping. Suppose that $f$ preserves $\rho$-orthogonality. The unit sphere $S$ in $X$ consists of four segments $\left[ \pm e_{1}, \pm e_{2}\right]$ where $e_{1}=$ $(1,0), e_{2}=(0,1)$. Let $x, y \in\left[e_{1}, e_{2}\right] \backslash\left\{e_{1}, e_{2}\right\}, x \neq y$. Then with some $t_{0} \in(0,1)$ we have $x+t(y-x) \in\left[e_{1}, e_{2}\right]$ for all $t \in\left[-t_{0}, t_{0}\right]$. Thus, $\|x+t(y-x)\|=1$ for $t \in\left[-t_{0}, t_{0}\right]$ and

$$
\begin{aligned}
\langle y-x \mid x\rangle_{g}= & \frac{1}{2}\left(\rho_{+}^{\prime}(x, y-x)+\rho_{-}^{\prime}(x, y-x)\right) \\
= & \frac{1}{2}\left(\lim _{t \rightarrow 0^{+}} \frac{\|x+t(y-x)\|^{2}-\|x\|^{2}}{2 t}\right. \\
& \left.+\lim _{t \rightarrow 0^{-}} \frac{\|x+t(y-x)\|^{2}-\|x\|^{2}}{2 t}\right) \\
= & 0 .
\end{aligned}
$$

It proves that $x \perp_{\rho} y-x$ and consequently, since $f$ preserves $\rho$-orthogonality, $f x \perp_{\rho}(f y-f x)$, hence $f x \perp_{\mathrm{B}}(f y-f x)$. The latter means that for each $\lambda \in \mathbb{R}$, $\|f x\| \leq\|f x+\lambda(f y-f x)\|$ thus, in particular, for $\lambda=1,\|f x\| \leq\|f y\|$. Interchanging the role of $x$ and $y$ in the above reasoning one gets $\|f y\| \leq\|f x\|$, hence $\|f x\|=\|f y\|$. This proves, that $f$ is constant on the segment $\left[e_{1}, e_{2}\right]$ and similarly one can prove the same for other segments. The continuity of $f$ yields that $\|f x\|$ is constant on the whole unit sphere $S$. Thus, $f$ is a similarity.

\section{Approximately $\rho$-orthogonality preserving mappings}

One can also consider linear mappings $f: X \rightarrow X$ that only approximately preserve the $\perp_{\rho}\left(\perp_{\rho_{ \pm}}\right)$orthogonality, i.e., which satisfy one of the following:

$$
x \perp_{\rho} y \Longrightarrow f x \perp_{\rho}^{\varepsilon} f y, \quad x, y \in X,
$$




$$
\begin{aligned}
& x \perp_{\rho_{+}} y \Longrightarrow f x \perp_{\rho_{+}}^{\varepsilon} f y, \quad x, y \in X, \\
& x \perp_{\rho_{-}} y \Longrightarrow f x \perp_{\rho_{-}}^{\varepsilon} f y, \quad x, y \in X .
\end{aligned}
$$

The latter two conditions are equivalent. Indeed, suppose that a linear mapping $f$ approximately preserves $\rho_{+}$-orthogonality. Let $x \perp_{\rho_{-}} y$. Equivalently, $-x \perp_{\rho_{+}} y$ and hence $-f x \perp_{\rho_{+}}^{\varepsilon} f y$. Thus, $f x \perp_{\rho_{-}}^{\varepsilon} f y$ which proves that $f$ approximately preserves $\rho_{-}$-orthogonality. The reverse implication can be proved analogously.

There are two natural questions. The first is to describe the given class of approximately orthogonality preserving mappings. The second is to answer the stability question: does each approximately orthogonality preserving mapping have to be approximated by an orthogonality preserving one. For inner product spaces with relations $\perp$ and $\perp^{\varepsilon}$ those questions were answered in $[7,8,20]$ and transferred to the realm of Hilbert $C^{*}$-modules in [14]. Similar investigations have been carried out by the authors in normed spaces for isosceles orthogonality (cf. [9]) and also by the second author for the Birkhoff and other orthogonalities (not yet published). Recently, the Birkhoff orthogonality preserving mappings and the problem of the stability of this property have been considered by Mojškerc and Turnšek [19].

In the case of a smooth space $X$ we have $\perp_{\rho}=\perp_{\mathrm{B}}$ and $\perp_{\rho}^{\varepsilon}=\perp_{\mathrm{B}}^{\varepsilon}$ for all $\varepsilon \in[0,1)$. Thus, if $X$ is smooth, the class of (approximate) $\rho$-orthogonality preserving linear mappings coincide with that of (approximate) Birkhoff orthogonality preserving ones and hence the stability problems for $\rho$ - and $B$ orthogonality preserving mappings are equivalent. It is known that for Birkhoff orthogonality the stability problem has an affirmative answer for some classes of spaces (including finite-dimensional ones) (cf. [19]).

The case of non-smooth spaces remains an open problem.

Open Access. This article is distributed under the terms of the Creative Commons Attribution Noncommercial License which permits any noncommercial use, distribution, and reproduction in any medium, provided the original author(s) and source are credited.

\section{References}

[1] Alsina, C., Sikorska, J., Santos Tomás, M.: On the equation of the $\rho$-orthogonal additivity. Commentat. Math. 47, 171-178 (2007)

[2] Alsina, C., Sikorska, J., Santos Tomás, M.: Norm Derivatives and Characterizations of Inner Product Spaces. World Scientific, Hackensack (2009)

[3] Amir, D.: Characterization of Inner Product Spaces. Birkhäuser Verlag, Basel (1986)

[4] Birkhoff, G.: Orthogonality in linear metric spaces. Duke Math. J. 1, 169-172 (1935)

[5] Blanco, A., Turnšek, A.: On maps that preserve orthogonality in normed spaces. Proc. R. Soc. Edinb. Sect. A 136, 709-716 (2006)

[6] Chmieliński, J.: On an $\varepsilon$-Birkhoff orthogonality. J. Inequal. Pure Appl. Math. 6(3) (2005) (Art. 79) 
[7] Chmieliński, J.: Linear mappings approximately preserving orthogonality. J. Math. Anal. Appl. 304, 158-169 (2005)

[8] Chmieliński, J.: Stability of the orthogonality preserving property in finite-dimensional inner product spaces. J. Math. Anal. Appl. 318, 433-443 (2006)

[9] Chmieliński, J., Wójcik, P.: Isosceles-orthogonality preserving property and its stability. Nonlinear Anal. 72, 1445-1453 (2010)

[10] Day, M.M.: Normed Linear Spaces. Springer, Berlin (1973)

[11] Dragomir, S.S.: On approximation of continuous linear functionals in normed linear spaces. An. Univ. Timişoara Ser. Ştiinţ. Mat. 29, 51-58 (1991)

[12] Dragomir, S.S.: Semi-Inner Products and Applications. Nova Science Publishers Inc, Hauppauge (2004)

[13] Giles, J.R.: Classes of semi-inner-product spaces. Trans. Am. Math. Soc. 129, 436446 (1967)

[14] Ilišević, D., Turnšek, A.: Approximately orthogonality preserving mappings on $C^{*}$-modules. J. Math. Anal. Appl. 341, 298-308 (2008)

[15] James, R.C.: Orthogonality and linear functionals in normed linear spaces. Trans. Am. Math. Soc. 61, 265-292 (1947)

[16] Koldobsky, A.: Operators preserving orthogonality are isometries. Proc. R. Soc. Edinb. Sect. A 123, 835-837 (1993)

[17] Lumer, G.: Semi-inner-product spaces. Trans. Am. Math. Soc. 100, 29-43 (1961)

[18] Miličić, P.M.: Sur la G-orthogonalité dans les espaces normés. Mat. Vesnik 39, 325334 (1987)

[19] Mojškerc, B., Turnšek, A.: Mappings approximately preserving orthogonality in normed spaces (manuscript)

[20] Turnšek, A.: On mappings approximately preserving orthogonality. J. Math. Anal. Appl. 336, 625-631 (2007)

Jacek Chmieliński

Instytut Matematyki

Uniwersytet Pedagogiczny w Krakowie

Podchorążych 2

30-084 Kraków

Poland

e-mail: jacek@up.krakow.pl

Paweł Wójcik

Instytut Matematyki

Uniwersytet Ślạski w Katowicach

Bankowa 14

40-007 Katowice

Poland

e-mail: pawelwojcikmmm@wp.pl

Received: February 27, 2010

Revised: July 21, 2010 